\title{
Passive wireless temperature sensor for high temperature applications with conductive surrounding
}

\author{
Dipl.-Ing. Tobias Dräger ${ }^{1}$, Dipl.-Ing. Josef Bernhard ${ }^{1}$ \\ ${ }^{1}$ Fraunhofer Institute for Integrated Circuits, Nordostpark 93, 90411 Nuremberg, Germany \\ \{tobias.draeger\}, \{josef.bernhard\}@iis.fraunhofer.de
}

\begin{abstract}
:
This paper presents a passive wireless temperature sensor designed for temperatures up to $200^{\circ} \mathrm{C}$ and to work on and in carbon fiber or other conductive materials. Similarly to low frequency (LF) radio frequency identification (RFID) transponders, the measurement circuit is powered by an external reader device through its magnetic field using coupled coil antennas. The sensor signal is modulated by means of load modulation and transmitted to the reader where it is detected and analyzed. The sensor has been fabricated and its accuracy has been tested and compared with common wired sensors. The presented sensor enables measurements inside the fiber material during its curing inside an oven in replacement or amends of common wired sensors.
\end{abstract}

Key words: passive wireless temperature sensor, carbon fiber, RFID.

\section{Introduction}

During fiber composite material production the curing of the fibers within an oven or autoclave with adequate pressure and temperature profile is one main step to achieve high performance fiber material structures. The needed process pressure and temperature can differ depending on the fiber material and structure application between normal room conditions and high values with several bar pressure and temperatures up to some hundred degrees [1], [2], [3]. During the curing process the structures temperature is monitored with the help of wired thermo elements on the structure form. For carbon fiber production it is common to cure several different parts within one autoclave run, as this production step is somehow the bottle neck of the whole manufacturing process. As a result, it is necessary to have as many temperature measurements as possible. However these only monitor the temperature value within the structures curing form, not within or on top of the fiber material itself. In order to guarantee a high quality output out of the cost intensive curing step, safety margins are added to the temperature profile to override the measurements uncertainty. In order to improve production quality, speed and cost, sensors that can operate wireless within the autoclave or even within the composite structure are a topic of research [1], [3].

This paper will first give some requirements for sensor systems and present an overview of commercial sensor solutions and other possible sensor principles. A solution using inductive coupling for energy transmission and load modulation for data transmission will be presented as a prototype with first sensor characterizations and integration tests performed wired and wireless.

\section{General sensor requirements}

In order to be used within a carbon fiber production process with autoclave, a sensor electronic should be able to operate at high temperatures $>120^{\circ} \mathrm{C}$ and several bar pressure. Furthermore, as carbon fiber is a conductive material and the autoclave itself and the structure forms are made from metal, the sensor has to deal with a metallic surrounding.

\section{Requirements wireless temperature sensors}

Beside optical temperature measurements there are two main solutions for wireless temperature sensors available: passive and active measurement systems. A commercial example for passive wireless temperature sensors are the so called surface acoustic wave (SAW) sensors. These sensors work with for example temperature depending reflections of a surface acoustic signal within a substrate. They can be connected to a high frequency antenna transforming the RF signal into surface acoustic wave signals and vice versa. A reader device sending a $2.45 \mathrm{GHz}$ signal receives the reflected signal being modified by the substrate behavior. These sensors, which are 
commercially available, can operate at high temperatures. Also it is possible to operate several sensors tuned to different resonance frequencies in parallel without signal collisions [7], [8], [9].

Active wireless sensor systems are often built as shown in Fig. 1.

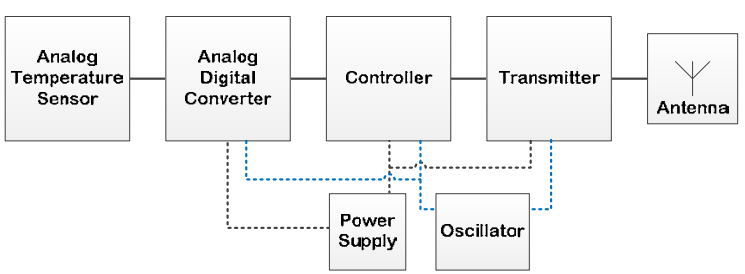

Fig. 1. Block diagram of common active wireless sensors.

Common active temperature systems consist of an analog temperature sensor and several circuits to convert this analogue sensor signal into a digital value and to transfer these values wireless to a receiver. Beside the accuracy of the sensor itself, the quality and resolution of the analog to digital (AD) conversion has strong influence on the system accuracy. For a stable and high resolution $A D$ conversion a stable supply and reference voltage as well as a stable oscillator frequency is mandatory.

Commercial wireless sensor systems guarantee stable and accurate values within their specifications, mainly for surrounding temperatures much lower than $85^{\circ} \mathrm{C}$. Challenging for higher operating temperatures are the power supply, a stable voltage regulation and frequency generation for such a system. Also, there can be a strong temperature influence on the antenna performance. For temperatures above $120^{\circ} \mathrm{C}$ only few components are available, as these temperatures are above common industrial and automotive standards.

As a result, the wireless temperature sensor within an autoclave should be able to operate with unstable voltage supply, without accurate oscillator frequency and with as few as possible complex components. Microcontrollers and analog to digital converters for signal processing should be avoided. In addition, the surrounding materials (autoclave, forms, carbon material) are conductive and will disturb any wireless transmission signal and detune the antennas.

\section{Investigated sensor principles}

In order to have alternatives to the above described common sensor solutions, other temperature sensitive circuits were investigated that could be able to deal with the fiber production process conditions:
- Operating at temperatures above $120^{\circ} \mathrm{C}$

- Working with unstable voltage levels

- Wireless read out possible within conductive surrounding

- Pressure insensitive

- Small size, especially small height

Two sensor principles where selected: the temperature dependence of the resonance behavior of a crystal oscillator and a temperature sensitive oscillator circuit.

\section{Crystal oscillator sensor}

In normal applications the temperature depending frequency shift of a crystal oscillator is undesired. In some cases high efforts are spent to regulate the oscillator circuit to provide a stable clock frequency within a specified temperature range [10], [11], [12]. Fig. 2 shows the principle circuit to measure the resonance frequency of a crystal.

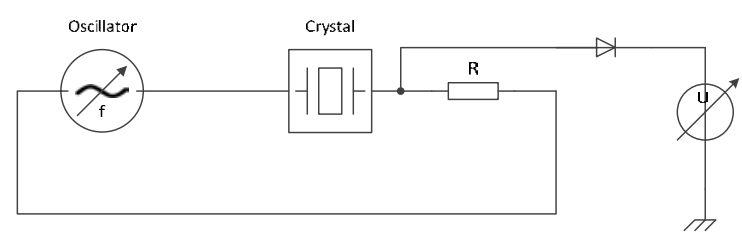

Fig. 2. Measurement set up with crystal.

The crystal is connected to an oscillator and a resistor in series. Because the crystal impedance changes from high to low when it is excited with its resonance frequency, the actual resonance frequency can easily be measured. By sweeping the frequency of an oscillator with constant output amplitude this impedance change can be measured as a voltage change between the crystal and the resistor R. For an easy measurement the voltage is converted from $A C$ to $D C$ with a diode. Fig. 3 shows an example for a wired measurement with an Epson HTS-206 Temperature sensing crystal with a nominal frequency at $40 \mathrm{kHz}$ [13].

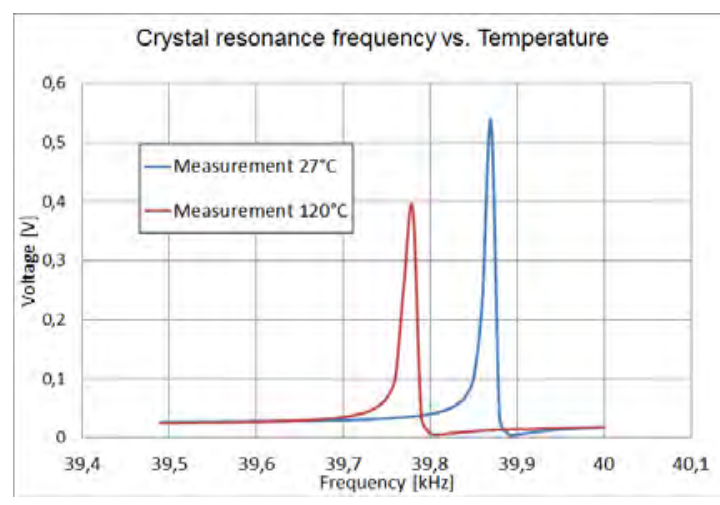

Fig. 3. Temperature effect on crystal resonance. 
In order to use this effect for a wireless temperature sensing, the circuit shown in Fig. 4 was built.

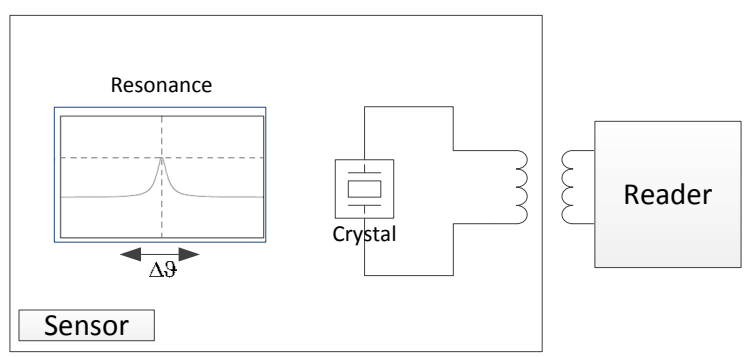

Fig. 4. Block diagram wireless crystal sensor.

This wireless solution is described in [14] and gives similar results in the lab as the wired solution. The reader transmits a magnetic field, sweeping its frequency and monitoring the power consumption of the transmitter which is influenced by the crystal impedance. Although this is a feasible solution for wireless sensing with high temperature and small size, the crystal is also pressure sensitive. For that reason this sensor principle was not further investigated.

\section{Temperature sensitive oscillator circuit}

A second investigated principle is that the sensor itself creates a temperature sensitive frequency by a simple "RC" oscillator circuit. The frequency is defined by a capacitor and a resistor connected to an operational amplifier as shown in Fig. 5.

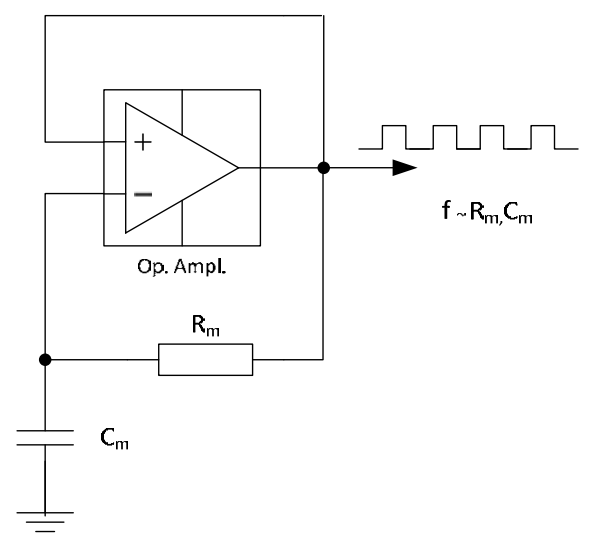

Fig. 5. Oscillator circuit with operational amplifier.

Using a temperature sensitive resistor like a NTC or PTC resistor the output of the circuit is a rectangle signal strongly temperature depending frequency.

Looking for a more simple circuit one suggestion is the so called "astable multivibrator" circuit, built with n-canal field effect transistors (N-FET) like shown in Fig. 6.

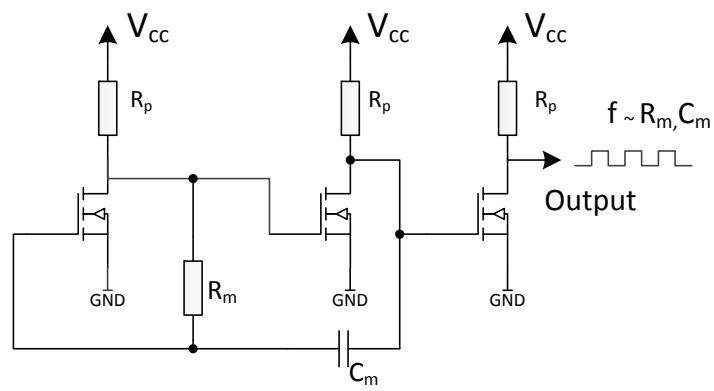

Fig. 6. Oscillator circuit with field effect transistors.

The circuit, built from three N-FETs, is oscillating at a frequency defined by $R_{m}$ and $C_{m}$. The circuit can oscillate between two stable states depending on whether the capacitor is being charged or discharged. This leads to a constant oscillation between the two stages caused by parasitic effects within the transistors, which do not switch exactly with the same behavior. As a result, a simulation of this circuit with ideal components will lead to no permanent oscillation signal at the output, but simulation with models of real transistors will do so.

After measurements using a NTC resistor for $R_{m}$ compared with circuits having "normal" fixed resistors at the same place, it could be seen that the temperature shift of standard components themselves is strong enough to generate a temperature depending output frequency. Simulation shows that this effect is even present within the transistors themselves, as the switching behavior is temperature dependent as given in equation (1) where $T$ is the temperature, $I_{D}$ is the drain current, $U_{G S}$ is the gate source voltage, $U_{t h}$ is the threshold voltage and $K$ is the transconductance coefficient. This behavior can also be seen in circuit simulation and literature [4], [5].

$\frac{1}{I_{D}} \cdot \frac{d I_{D}}{d T}=\frac{1}{K} \cdot \frac{d K}{d T}-\frac{2}{U_{G S}-U_{t h}} \frac{d U_{t h}}{d T}$

To determine the influence of the transistors Fig. 7 shows the corresponding output signal of a simulated circuit as shown in Fig. 6, but with ideal resistors and capacitors and the model of the Si1012R/X n-canal FET provided by Vishay [15]. 


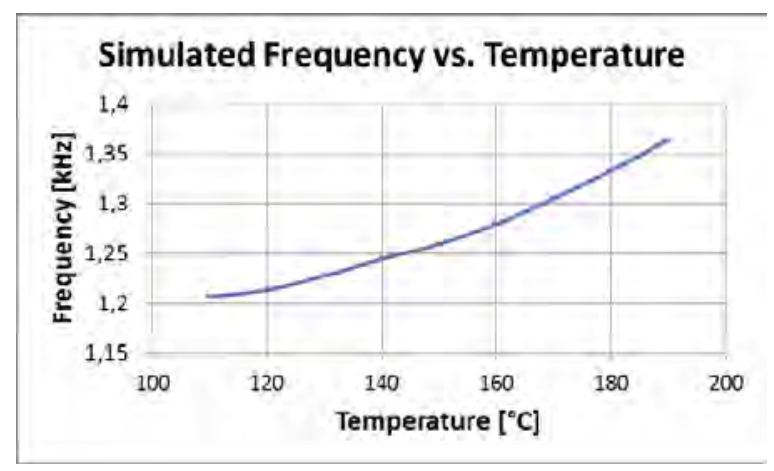

Fig. 7. Simulated frequency behavior over temperature.

This simulation result was validated by measurements in a feutron climate and humidity test chamber TPK 190.

\section{Wireless circuit with temperature sensitive oscillator sensor circuit}

In order to read out the circuit output frequency wireless two additional challenges are left. The circuit has to be powered with a DC voltage supply for proper operation and the output signal has to be transmitted wireless to a read out station.

\section{Wireless power transmission}

The power supply can be solved by means of resonant coupled coils, forming a week coupled air gap transformer for inductive wireless power transmission. As shown in Fig. 8, the read out unit generates through a transmission coil an alternating magnetic field that induces a voltage within the sensor coil antenna. This alternating voltage is converted into $\mathrm{DC}$ and regulated by a simple circuit built with a bipolar transistor and a zener diode to provide a more or less stable voltage level for the sensor circuit.

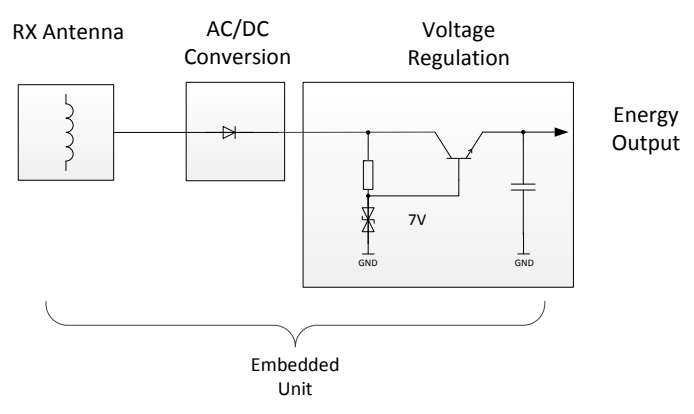

Fig. 8 Block diagram wireless power transmission.

This voltage regulation is very similar to a simple linear voltage regulator but built from very few components. The result is a stable supply voltage for the sensor circuit that is, with carefully chosen components, not too sensitive to the surrounding temperature. Simulations show that the sensor output signal is not influenced by the voltage regulation over temperature for the investigated temperature values between 100 and $180^{\circ} \mathrm{C}$.

\section{Wireless sensor signal transmission}

As the sensor circuit is supplied with energy through inductive coupled coils, the transmission of the sensor signals can also use these coils by means of load modulation. Commonly known from inductive coupled RFID systems, the embedded circuit adds an additional modulation load to its antenna. Doing this with a certain frequency leads to an alternating change of power consumption of the embedded circuit and in result to a modulation of the field strength that can be detected and demodulated by an external unit (Fig. 9).

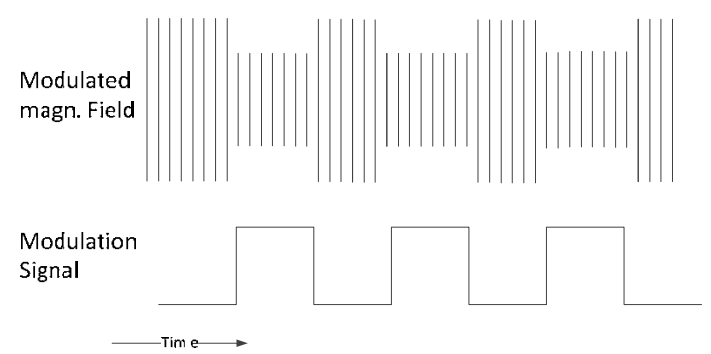

Fig. 9. Principle drawing load modulation.

In this case the rectangle output signal of the sensor circuit can be used directly as a modulation signal, using the temperature influenced rectangle signal to control a modulation transistor. This leads to a direct transmission of the sensor signal to an external unit without digitalization or signal processing within the embedded sensor circuit. The complete circuit schematic is shown in Fig. 10 with the voltage conversion and regulation (1), the sensor circuit 2, the load modulation circuit 3 and the sensor coupled coil 6 .

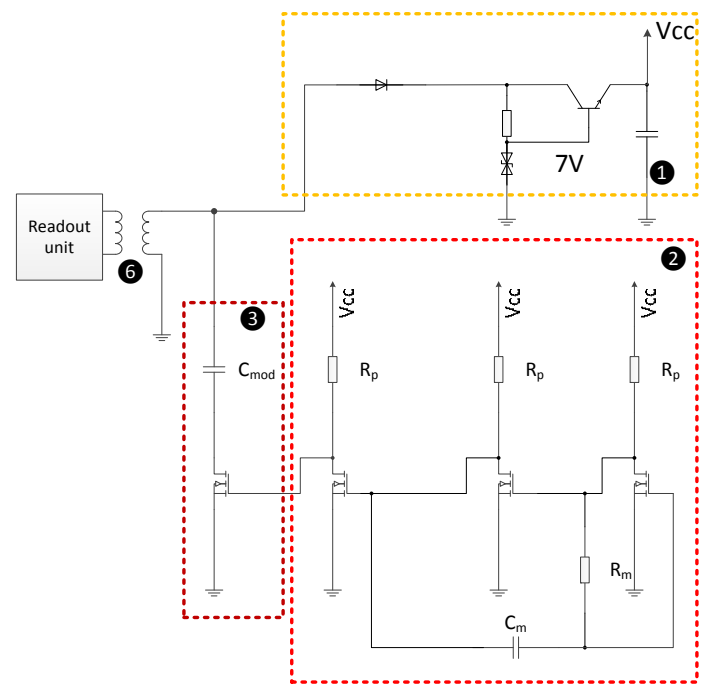

Fig. 10. Complete sensor circuit with read out unit. 


\section{Prototype pcb}

For further measurements a high temperature pcb with teflon core was designed. The small $18 \mathrm{~mm}$ diameter pcb (Fig. 11) contains beside the already shown schematic blocks (123) in addition an RFID transponder (5) for identification of the different pcbs during measurements and a PT1000 resistor (4) to measure temperature directly on the pcb during the sensor circuit characterization.

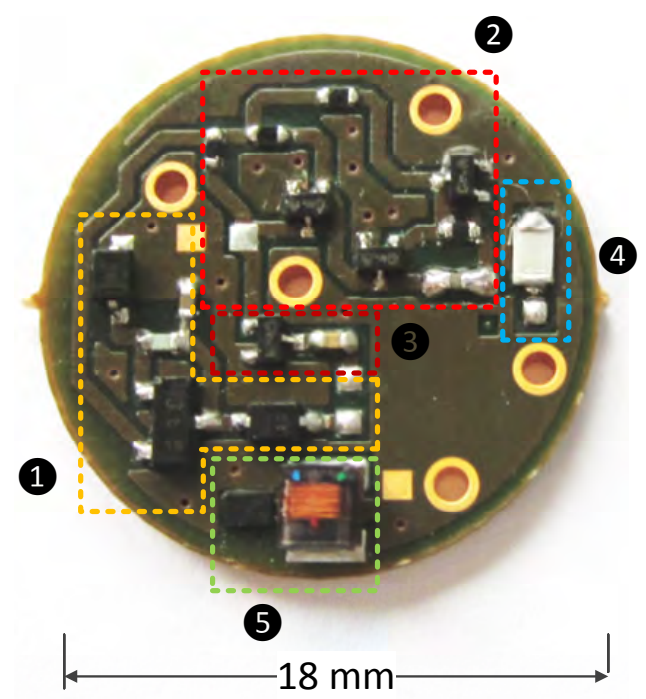

Fig. 11. Prototype sensor pcb.

This prototype pcb was measured inside a climate chamber as well as inside a laboratory autoclave similar to those used for the production of fiber materials. First measurement results are shown in Fig. 12.

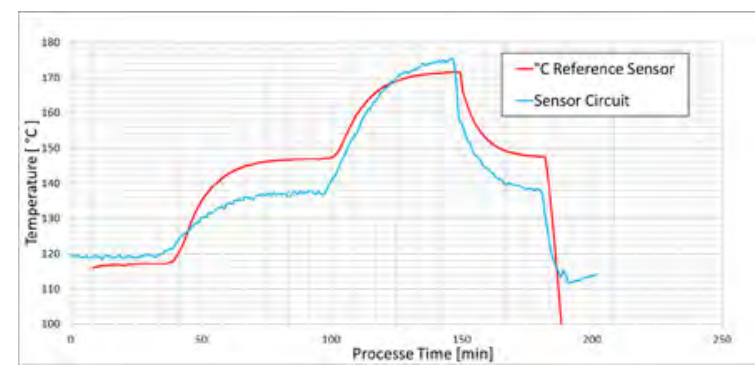

Fig. 12. Comparison sensor output vs. reference sensor.

These results were converted from frequency measurements (sensor output) into temperature values with a two point calibration at $125^{\circ} \mathrm{C}$ and $170^{\circ} \mathrm{C}$. With this calibration a comparison to a reference sensor is possible. The sensor values follow the reference shape but it can be seen that a more accurate polynomial approximation is needed in the future.

\section{Wireless sensor for conductive surrounding}

The wireless version of the sensor board was applied on peel-ply material with a sewed coil for LF (125 kHz, Antenna produced by Tajima
$\mathrm{GmbH}$ ) inductive coupling (Fig. 13). With this frequency a later application of the sensor in proximity to conductive material as for example carbon fiber is possible [6]. The use of peel-ply guarantees a good compatibility for the fiber material production process and a simple removing of the sensor after curing.

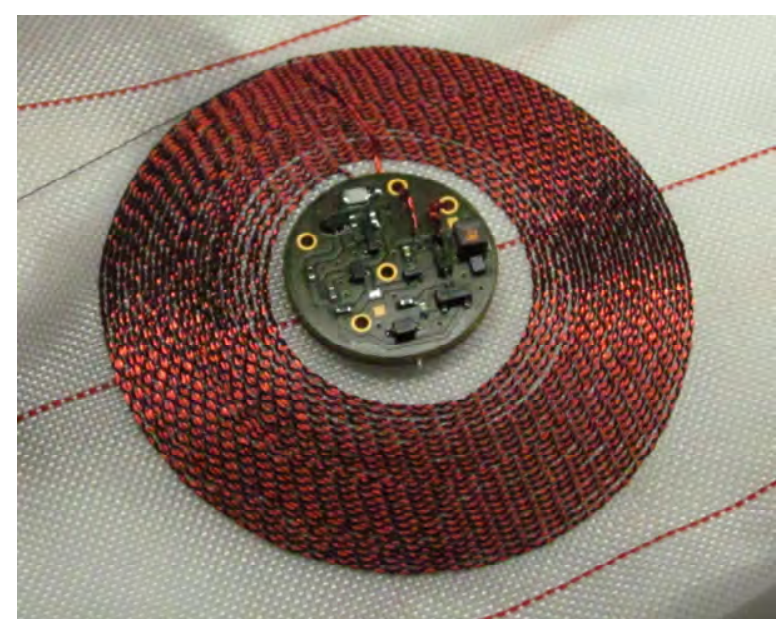

Fig. 13. Wireless version with sewed antenna on peel-ply material.

\section{Summary, conclusions and future work}

This paper presented a new approach for a passive wireless temperature sensor for high temperature applications with conductive surrounding. After mentioning the motivation for the sensors development common commercial wireless sensor principles have been introduced. In addition two alternative sensor principles for passive temperature measurements were presented. The most promising principle is a temperature sensitive oscillator circuit, which is investigated and tested with different implementations as well as with a prototype pcb. This sensor principle seems to be adequate for several fiber material production processes. First tests show that the results can match reference measurements. In the future this sensor circuit will be tested and investigated in detail to get reliable data about its performance and accuracy. Also it is planned to embed the circuit into carbon fiber material. A simulation of the circuit for a common CMOS integration process showed that an integrated circuit design of the sensor circuit is possible with acceptable performance using a two point calibration.

\section{Acknowledgments}

We extend our sincere thanks to the Bavarian Research Foundation (Bayerische Forschungsstiftung) for the funding of our research. The results presented in this paper 
have been achieved within the scope of the project „Application of RFID in the production of fiber reinforced plastics" (Einsatz von RFID bei der Herstellung von Faserverbundwerkstoffen, AZ-835-08).

\section{References}

[1] Fleming, M.; Ziegmann, G.: Faserverbundbauweisen - Fasern und Matrices, 1995, Springer, Berlin

[2] J. Zettler, Prof. G. Reinhart, T. Philipp, J. Arent, T. Dräger, C. Grabowski, Influence of Integrated RFID Transponders on Structural Behavior of Carbon Fiber-Reinforced Plastics, 6th International Conference "Supply on the wings" 2011

[3] AVK - Industrievereinigung Verstärkte Kunststoffe E.V. (HRSG.) 2010 AVK - Industrievereinigung Verstärkte Kunststoffe e.V. (Hrsg.): Handbuch Faserverbundkunststoffe. 3., vollst. überarb. Aufl. Wiesbaden: Vieweg + Teubner 2010. ISBN: 9783-8348-0881-3

[4] Tietze, Schenk, Halbleiter-Schaltungstechnik, 12. Auflage, Springer Verlag 2002

[5] Salah, Khachroumi, Morel, Sensors 2012, 10, 388-399; doi:10.3390/s100100388

[6] Bernhard, Josef; Draeger, Tobias; Grabowski, Christoph; Sotriffer, Ingo; Philipp, Tobias; , "Integrating RFID in Fibre-Reinforced Plastics," RFID SysTech 2011; 7th European Workshop on Smart Objects: Systems, Technologies and Applications; Proceedings of , vol., no., pp.1-7, 17-18 May 2011

[7] Wen Liu; Bing Tan; Yiwen Gong; , "SAW temperature sensors system for smart electric grid," Ultrasonics Symposium (IUS), 2010 IEEE, vol., no., pp.756-759, 11-14 Oct. 2010 doi: 10.1109/ULTSYM.2010.5935725

[8] Reindl, L.; Shrena, I.; Kenshil, S.; Peter, R.; , "Wireless measurement of temperature using surface acoustic waves sensors," Frequency Control Symposium and PDA Exhibition Jointly with the 17th European Frequency and Time Forum, 2003. Proceedings of the 2003 IEEE International , vol., no., pp. 935- 941, 4-8 May 2003doi: 10.1109/FREQ.2003.1275216

[9] CTR AG, Wireless sensors for extreme conditions, Online, http://www.ctr.at/en/r-dtechnologies/wireless-sensors.html

[10] Hewlett Packard Application Note 200-2, Fundamentals of Quartz Oscillators, 1997

[11] Cardinal Components Inc. Applications Brief No. A.N. 1006, Clock Oscillator Stability

[12] VDI/VDE 3511 Blatt 1, Technische Temperaturmessungen - Grundlagen und Übersicht über besondere Temperaturmeßverfahren, 1996-03

[13] Datasheet Temperature Sensing Crystal HTS206, Online http://www.eea.epson.com
[14] Diplomarbeit David Eisele, Entwicklung eines induktiv gekoppelten Quarztemperatursensors, Lehrstuhl für Elektrische Mess- und Prüfverfahren Albert-Ludwigs-Universität Freiburg

[15] Vishay Electronic $\mathrm{GmbH}$, Product Information nChannel 1.8 V MOSFET Si1012R/X, Online http://www.vishay.com/mosfets/list/product71166/ 